\title{
Comparison of Four SARS-CoV-2 Neutralization Assays
}

\author{
Lydia Riepler ${ }^{\dagger}$, Annika Rössler ${ }^{\dagger}$, Albert Falch, André Volland, Wegene Borena $\mathbb{C}^{\circledR}$, Dorothee von Laer \\ and Janine Kimpel *(D)
}

check for

updates

Citation: Riepler, L.; Rössler, A.; Falch, A.; Volland, A.; Borena, W.; von Laer, D.; Kimpel, J. Comparison of Four SARS-CoV-2 Neutralization Assays. Vaccines 2021, 9, 13. https:// dx.doi.org/10.3390/vaccines 9010013

Received: 27 November 2020 Accepted: 20 December 2020 Published: 28 December 2020

Publisher's Note: MDPI stays neutral with regard to jurisdictional claims in published maps and institutional affiliations.

Copyright: () 2020 by the authors. Licensee MDPI, Basel, Switzerland. This article is an open access article distributed under the terms and conditions of the Creative Commons Attribution (CC BY) license (https: / / creativecommons.org/ licenses/by/4.0/).
Department of Hygiene, Microbiology and Public Health, Institute of Virology, Medical University of Innsbruck, 6020 Innsbruck, Austria; lydia.riepler@i-med.ac.at (L.R.); Annika.Roessler@i-med.ac.at (A.R.); Albert.Falch@i-med.ac.at (A.F.); andre.volland@i-med.ac.at (A.V.); Wegene.Borena@i-med.ac.at (W.B.); dorothee.von-laer@i-med.ac.at (D.v.L.)

* Correspondence: Janine.kimpel@i-med.ac.at; Tel.: +43-512-9003-71725

+ These authors contributed equally to this work.

\begin{abstract}
Neutralizing antibodies are a major correlate of protection for many viruses including the novel coronavirus SARS-CoV-2. Thus, vaccine candidates should potently induce neutralizing antibodies to render effective protection from infection. A variety of in vitro assays for the detection of SARS-CoV-2 neutralizing antibodies has been described. However, validation of the different assays against each other is important to allow comparison of different studies. Here, we compared four different SARS-CoV-2 neutralization assays using the same set of patient samples. Two assays used replication competent SARS-CoV-2, a focus forming assay and a $\mathrm{TCID}_{50}$-based assay, while the other two assays used replication defective lentiviral or vesicular stomatitis virus (VSV)-based particles pseudotyped with SARS-CoV-2 spike. All assays were robust and produced highly reproducible neutralization titers. Titers of neutralizing antibodies correlated well between the different assays and with the titers of SARS-CoV-2 S-protein binding antibodies detected in an ELISA. Our study showed that commonly used SARS-CoV-2 neutralization assays are robust and that results obtained with different assays are comparable.
\end{abstract}

Keywords: SARS-CoV-2; neutralizing antibodies; neutralization assay; pseudotype virus

\section{Introduction}

Since the first cases of SARS-CoV-2 infection have been reported in Wuhan in December 2019 the virus has spread throughout the world and caused a severe pandemic. Up to now more than 76 million infections with SARS-CoV-2 and more than 1.6 million deaths have been reported [1]. In order to control the pandemic, identifying and isolating infected patients is a crucial step. Usually, acutely infected persons are identified via qPCR testing of respiratory swab samples.

During a SARS-CoV-2 infection, patients develop adaptive immune responses against the virus. The knowledge of past infections, i.e., the serostatus of individuals or of a population, is important information as it will be an indicator for immunity. Reliable testing for the serostatus will also be important when judging the efficacy of potential vaccine candidates, the need for revaccination, or selection of plasma donors for therapy with plasma from convalescent patients. There are a great number of immunoassays such as ELISAs available for detection of SARS-CoV-2-binding antibodies [2]. Often, antibodies against the spike protein $\mathrm{S}$ or the nucleoprotein $\mathrm{N}$ are detected. Many of these assays have a high specificity and sensitivity, especially when a combination of different assays is used [3,4]. Immunoassays such as ELISAs have the advantage that they are easy to standardize and validate. Additionally, high throughput analysis of samples is possible. However, such immunoassays do not provide information on the functionality of the antibodies detected. Especially neutralizing antibodies are considered to be an important factor for immunity and potentially for the clearance of the virus in the infected 
individuals. The titers and kinetics of antibody production during a SARS-CoV-2 infection vary considerably and might depend on the severity of disease [5,6]. Additionally, it is not yet completely clear how long SARS-CoV-2 neutralizing antibodies last and consequently, if they can mediate long-term protection [7]. Recently, the first reinfections of convalescent patients have been reported [8,9]. Although, some studies have shown a correlation of binding and neutralizing antibodies $[4,10,11]$, neutralizing antibody assays are still a gold standard to judge the immunity of a patient.

Different types of SARS-CoV-2 neutralization assays have been described using either replication competent SARS-CoV-2 virus [12,13] or SARS-CoV-2 spike protein (S) pseudotyped lentiviral [14] or vesicular stomatitis virus (VSV)-based particles [15-17]. Assays with replication competent SARS-CoV-2 isolates are normally either plaque reduction/focus forming assays or $\mathrm{TCID}_{50}$-based assays. However, they have the disadvantage that they require biosafety level (BSL)-3 laboratories and are often labor intense. On the other hand, assays using replication-defective pseudotyped viral particles can be performed under BSL-1 or BSL-2 conditions. However, validation of these assays against assays using replication competent SARS-CoV-2 is necessary. As all neutralization assays require living cells, they are more difficult to standardize than ELISAs and, therefore, testing of robustness of these assays is a crucial step.

Many SARS-CoV-2 studies, such as epidemiological studies, vaccine efficacy trials, trials to analyze the efficacy of plasma from COVID-19 convalescent patients as a treatment option, etc., determine neutralizing antibody titers. However, it is often difficult to compare results as different assays are used. Therefore, a thorough comparison of assays is necessary. Here, we optimize four different SARS-CoV-2 neutralization assays, two assays using replication competent SARS-CoV-2, one assay with S pseudotyped lentiviral particles, and one assay with S pseudotyped replication defective VSV particles, and determine interassay variability. Further, we compare all assays using the same standard set of COVID-19 convalescent patients.

\section{Materials and Methods}

\subsection{Cell Lines and Plasmids}

An expression plasmid encoding a C-terminally truncated, codon-optimized spike glycoprotein (pCG1-SARS-2-S $\Delta 18$ ) and a lentiviral vector encoding TMPRSS2 were kindly provided by Dr. Markus Hoffmann and Dr. Stefan Pöhlmann $[16,18]$. The truncated SARS-CoV-2 spike protein or human ACE2 were subcloned in a lentiviral vector (pLenti CMVie-IRES-BlastR, addgene \#119863) for generation of stable cell lines. 293T cells (ATCC) and derivates were cultured in high glucose Dulbecco's Modified Eagle Medium (Merck, Darmstadt, Germany) supplemented with 10\% fetal bovine serum (FBS, Gibco, Carlsbad, CA, USA), 2\% L-Glutamin (200 mM, Gibco), 1\% Penicillin-Streptomycin (10.000 U/mL, Gibco), 1\% MEM Non-Essential Amino Acids Solution (100×, Gibco), and 1\% Sodium Pyruvate (100 mM, Gibco). BHK-21 cells (American Type Culture Collection, Manassas, VA, USA) were maintained in Glasgow minimum essential medium (GMEM) (Gibco) supplemented with 10\% FBS, 5\% tryptose phosphate broth (Gibco), and 1\% PenicillinStreptomycin. Serum adapted Vero (AC-free) (ECACC 08011101) cells were cultured in high glucose Dulbecco's Modified Eagle Medium (Merck) supplemented with 10\% FBS, 2\% L-Glutamin, and 1\% Penicillin-Streptomycin, for infection experiments with SARS-CoV-2 medium with 2\% FBS was used. For Vero cells stably expressing TMPRSS2 (kindly provided by Dr. Markus Hoffmann and Dr. Stefan Pöhlmann) [16], medium was additionally supplemented with $10 \mu \mathrm{g} / \mathrm{mL}$ blasticidin. Lentiviral vectors were produced as described previously and used to generate ACE2 or S stable cell lines [19]. Cells were selected with $10 \mu \mathrm{g} / \mathrm{mL}$ blasticidin or $500 \mu \mathrm{g} / \mathrm{mL}$ hygromycin, depending on the resistance cassette of the lentiviral vector. All cell lines were cultured in humidified incubators at $37^{\circ} \mathrm{C}$ and $5 \% \mathrm{CO}_{2}$. 


\subsection{Plasma Samples and Binding Antibody Testing}

Plasma samples were obtained from SARS-CoV-2 convalescent or naïve donors. Plasma samples were heat inactivated at $56{ }^{\circ} \mathrm{C}$ for $30 \mathrm{~min}$ and subsequently centrifuged for $5 \mathrm{~min}$ at $8000 \mathrm{rpm}$ in a tabletop centrifuge. To determine titers of SARS-CoV-2 binding antibodies a commercially available anti-SARS-CoV-2-IgA and -IgG ELISA (Euroimmun, Lübeck, Germany) using the fully automated four-plate benchtop instrument Immunomat ${ }^{\mathrm{TM}}$ (Virion/Serion, Würzburg, Germany) for detection of anti-S IgA and anti-S IgG and an Abbott SARS-CoV-2 IgG immunoassay on the ARCHITECT i2000SR system (Abbott, Chicago, IL, USA) for detection of anti-N IgG antibodies were used. Cut-off values for all assays were used according to manufactures recommendations. For anti-S IgA and anti-S IgG antibodies an optical density (OD) $<0.8$ was interpreted as negative, an OD between 0.8 and 1.1 as borderline, and an OD $>1.1$ as positive result. The anti-N IgG immunoassay was interpreted as positive for relative light units (RLU) $>1.4$.

\subsection{Viruses}

The human replication competent SARS-CoV-2 was isolated from a respiratory swab sample from a qPCR positive tested patient at Medical University of Innsbruck (isolate 1.2) on Vero cells expressing TMPRSS2. The virus was propagated on Vero-TMPRSS2 cells. Cells were infected with an MOI of 0.001 and supernatant was harvested $70 \mathrm{~h}$ after infection, clarified by $0.45 \mu \mathrm{m}$ filtration, and frozen at $-80^{\circ} \mathrm{C}$. Viral titers were determined using focus forming or $\mathrm{TCID}_{50}$ assay as described below. VSV $\Delta \mathrm{G}$ viruses were generated similarly as previously described [20]. Shortly, VSV $\Delta$ G viruses with GFP or luciferase as marker gene were produced on BHK-21 cells stably expressing LCMV GP. Viruses were titrated via TCID 50 assay on BHK-21-LCMV-GP cells. For generation of SARS-CoV-2 S pseudotyped particles, subconfluent 293T cells stably expressing a C-terminally truncated version of $\mathrm{S}$ were infected with an MOI of 3 of the VSV $\Delta \mathrm{G}$-GP seed stock. Then, $1.5 \mathrm{~h}$ after infection, inoculum was removed, cells were washed once with PBS, and fresh medium supplemented with an LCMV GP-neutralizing rabbit serum (1:200, in-house produced) was added. A total of $36 \mathrm{~h}$ after infection, supernatant was collected, clarified via $0.45 \mu \mathrm{m}$ filtration, and stored in single-use aliquots at $-80^{\circ} \mathrm{C}$. Lentiviral particles were generated as described previously [19]. Briefly, $10 \mathrm{~cm}$ dishes of $293 \mathrm{~T}$ cells were transfected with $7.5 \mu \mathrm{g}$ lentiviral transfer vector plasmid (encoding GFP, ACE2, TMPRSS2, or spike), $12.5 \mu \mathrm{g}$ lentiviral Gag/Pol plasmid pCMV-dR8.91, $2 \mu \mathrm{g}$ of VSV-G, or $4 \mu \mathrm{g}$ of SARS-CoV-2 S encoding plasmid. Prior to transfection medium was replaced by complete medium without FBS. Then, $6 \mathrm{~h}$ after transfection, medium was exchanged, and complete medium was added. Supernatants were collected $24-48 \mathrm{~h}$ after transfection, clarified via $0.45 \mu \mathrm{m}$ filtration, and stored at $-80^{\circ} \mathrm{C}$ or directly used for generation of stable cell lines.

\subsection{Western Blotting}

Western blotting was performed as previously described [21]. Proteins were detected using an anti-ACE2 antibody (R\&D Systems, clone 171606, 1:5000 diluted) or an anti-tubulin antibody (Sigma, clone B-5-1-2, 1:4000 diluted) and horseradish peroxidase-conjugated secondary antibodies (mouse IgG-specific antibody from goat, Dianova, Hamburg, Germany).

\subsection{Focus Forming Assay with Replication Competent SARS-CoV-2}

For titration of virus using the focus forming assay, 96-wells containing $90 \%$ confluent Vero-TMPRSS2 cells $\left(1.8 \times 10^{4}\right.$ cells seeded one day prior to the assay) were infected in duplicates with $50 \mu \mathrm{L}$ of serial half-logarithmic diluted virus for $1 \mathrm{~h}$ at $37^{\circ} \mathrm{C}$. Subsequently, inoculum was removed, cells were washed once, and cultured for further $9 \mathrm{~h}$ in $100 \mu \mathrm{L}$ fresh medium. After fixation for 5 min with $96 \%$ ethanol, cells were stained using serum from a SARS-CoV-2 recovered patient and a horse radish peroxidase (HRPO)-conjugated antihuman secondary antibody. The signal was developed using a 3-amino-9-ethylcabazole (AEC) substrate and the number of infected cells were counted using an ImmunoSpot S6 Ultra-V reader and CTL analyzer BioSpot ${ }^{\circledR} 5.0$ software (CTL Europe GmbH, Bonn, 
Germany). Instrument settings as specified in Supplementary Table S1 were used for automatic counting. For quality control, counted well size was set to $96 \%$ to avoid background signal at the edge of the wells. Fibers were removed using the fiber removal function or manually. For neutralization assays, plasma samples were 4 -fold diluted in duplicate samples in medium containing $2 \%$ FCS, starting dilution 1:16. Plasma dilutions were mixed with an equal volume of SARS-CoV-2 virus resulting in 100-200 infected cells in non-neutralized wells. Plasma/virus mixes were incubated for $1 \mathrm{~h}$ at $37^{\circ} \mathrm{C}$ and subsequently transferred to 96 -wells containing $90 \%$ confluent Vero-TMPRSS2 seeded the day before. Cells were infected with virus for $1 \mathrm{~h}$ at $37^{\circ} \mathrm{C}$, subsequently washed once, and cultured for further $5,7,8,9,11$, or $13 \mathrm{~h}$ in fresh medium prior fixation and staining as described above. The $50 \%$ neutralization titers were calculated as highest plasma dilution where mean infection of duplicate samples was lower than $50 \%$ of the mean (quadruplicate samples) of control wells without plasma.

\section{6. $T C I D_{50}$-Based Assay}

To determine $\mathrm{TCID}_{50}$ titer, virus stocks were diluted serial 10-fold and subconfluent Vero/TMPRSS2-ACE2 cells were infected in quadruplicate samples. Plates were incubated for $48 \mathrm{~h}$ at $37^{\circ} \mathrm{C}$, subsequently, CPE positive wells were counted, and virus titer was calculated according to the Sperman-Kaerber formula. For neutralization assays, plasma samples were 4 -fold diluted in quadruplicates and mixed with an equal volume of SARS-CoV-2 virus corresponding to $100 \mathrm{TCID}_{50}$ per sample. After $1 \mathrm{~h}$ preincubation at $37^{\circ} \mathrm{C}$, plasma/virus mixes were transferred to 96 -wells containing $1 \times 10^{4}$ adherent Vero/TMPRSS2-ACE2 seeded the day before. Cells were incubated at $37^{\circ} \mathrm{C}$ for $48 \mathrm{~h}$ prior evaluation of CPE via microscope. Virus dilution used for infection was retitrated in each experiment. Neutralization titers of plasma samples were determined by the highest plasma dilution protecting $50 \%$ of the infected wells.

\subsection{VSV-Based Assay}

For titration, VSV $\Delta$ G-S stocks were diluted half-logarithmic in medium and subsequently used to infect subconfluent 293T-ACE2 cells seeded 1 day before. Titrations were analyzed $\approx 16 \mathrm{~h}$ after infection. For GFP-containing virus, medium was removed, and plates were analyzed using an ImmunoSpot S6 Ultra-V reader and FluoroSpot software (CTL Europe GmbH, Bonn, Germany). Instrument settings as specified in Supplementary Table S2 were used for automatic counting. For quality control, counted well size was set to $96 \%$ to avoid background signal at the edge of the wells due to remaining liquid. Fibers were removed using the fiber removal function or manually. For luciferase-containing virus, medium was aspirated, in-house produced lysis buffer (0.5\% IGEPAL CA-630, Sigma and $100 \mathrm{mM} \mathrm{NaCl}$ in $10 \mathrm{mM}$ Tris $\mathrm{HCl}, \mathrm{pH}=7.4$ ) was added, and $30 \mu \mathrm{L}$ cell lysate was transferred to white 96-well plates. Then, $50 \mu \mathrm{L}$ of $50 \mathrm{mg} / \mathrm{mL}$ VivoGlo luciferin (Promega $\mathrm{GmbH}$, Mannheim, Germany) were added and plates were analyzed immediately using a GloMax ${ }^{\circledR}$ Discover Microplate Reader (Promega GmbH, Mannheim, Germany) and Biotek Gen5 ${ }^{\mathrm{TM}}$ software. For neutralization assays, plasma samples were 4-fold diluted in medium and mixed with VSV $\Delta$ G-S virus with desired reporter gene. After $1 \mathrm{~h}$ preincubation mixes were used to infect subconfluent 293T-ACE2 cells. Plates were analyzed after $\approx 16 \mathrm{~h}$ as described above depending on the reporter gene. The $50 \%$ neutralization titers were calculated as highest plasma dilution where mean infection of duplicate samples was lower than $50 \%$ of the mean (quadruplicate samples) of control wells without plasma.

\subsection{Lentiviral Particle-Based Assay}

Lentiviral particles pseudotyped with SARS-CoV-2 S and with GFP as reporter gene were produced as described above. For titration, virus was half-logarithmic diluted in medium and subsequently used to infect subconfluent 293T-ACE2 cells in 96-wells. Two days after infection, medium was removed and the number of GFP positive cells was determined using an ImmunoSpot S6 Ultra-V reader and FluoroSpot software (CTL Europe 
$\mathrm{GmbH}$, Bonn, Germany). For neutralization assays, plasma was 4-fold serially diluted in medium and mixed with lentiviral particles resulting in $\approx 100-200$ GFP positive cells in virus only control wells. After $1 \mathrm{~h}$ of preincubation, mixes were used to infect subconfluent 293T-ACE2 cells and cells were incubated for 2 days. The 50\% neutralization titers were calculated as highest plasma dilution where mean infection of duplicate samples was lower than $50 \%$ of the mean (quadruplicate samples) of control wells without plasma.

\subsection{Statistics}

Correlation analysis was performed using GraphPad Prism 8.2.0 (GraphPad Software, Inc., La Jolla, CA, USA). Not all data sets had a Gaussian distribution. Therefore, a nonparametric Spearman correlation was computed with a two-tailed $p$ value and a $95 \%$ confidence interval.

\section{Results}

In this study, we established four different neutralization assays for SARS-CoV-2, a focus forming assay without virus spread and a TCID 50 assay with multiple full virus replication cycles and spread using replication competent SARS-CoV-2 and two pseudovirus assays using replication defective VSV or lentiviral particles pseudotyped with SARS-CoV-2 spike, and compared neutralization titers obtained with these assays using a set of samples from SARS-CoV-2 convalescent patients and healthy donors.

In a first step, we characterized the set of patients using different immunoassays to determine binding antibodies to SARS-CoV-2. Table 1 summarizes titers of anti-S IgG, anti-S IgA, and anti-N IgG antibodies. Our cohort comprised samples negative in all three assays (P4 and P11), samples with discrepant results in the immunoassays (P2 and P7 with anti-S IgG positive but anti-S IgA and anti-N IgG negative results), and samples that were positive in all three immunoassays. We included both weakly positive and strongly positive samples. This set of 11 patients was subsequently analyzed in all four neutralization assays.

Table 1. Titers of binding antibodies.

\begin{tabular}{cccc}
\hline Patient & Anti-S IgG $\$$ & Anti-S IgA $\$$ & Anti-N IgG \\
\hline P1 & 3.669 (positive) & 1.345 (positive) & 2.90 (positive) \\
P2 & 1.493 (positive) & 0.275 (negative) & 1.10 (negative) \\
P3 & 3.103 (positive) & 1.317 (positive) & 2.60 (positive) \\
P4 & 0.715 (negative) & 0.375 (negative) & 0.00 (negative) \\
P5 & 2.275 (positive) & 0.827 (borderline) & 2.14 (positive) \\
P6 & 3.970 (positive) & 1.130 (positive) & 3.00 (positive) \\
P7 & 1.183 (positive) & 0.523 (negative) & 0.81 (negative) \\
P8 & 5.298 (positive) & 8.745 (positive) & 4.20 (positive) \\
P9 & 1.722 (positive) & 1.283 (positive) & 2.30 (positive) \\
P10 & 7.180 (positive) & 6.325 (positive) & 7.54 (positive) \\
P11 & 0.156 (negative) & 0.558 (negative) & 0.07 (negative) \\
\hline
\end{tabular}

$\S<0.8$ negative; $0.8-1.1$ borderline; $>1.1$ positive; ${ }^{\$}>1.4$ positive.

\subsection{Focus Forming Unit-Assay Using Replication Competent SARS-CoV-2}

For the focus forming assay we preincubated replication competent SARS-CoV-2 for $1 \mathrm{~h}$ with different dilutions of patient sera and subsequently infected highly susceptible Vero cells expressing TMPRSS2. After different time periods, cells were fixed and stained with the serum from a SARS-CoV-2 convalescent patient. In a first step, we determined the optimal infection time to achieve the maximal number of infected single cells before the virus started to spread and foci with several cells started to appear. As we counted infected cells automatically using an immunospot reader the goal was to obtain individual infected cells but no clusters. Figure 1a shows exemplary pictures of infected cells after different infection times. After $6 \mathrm{~h}$, first infected cells were visible via immunostaining. However, the signal was weak at this time point. The intensity of the staining increased over time. After $12 \mathrm{~h}$, first virus spread, and clusters of infected cells were visible. To analyze the effect of the different incubation times on neutralizing antibody titers we analyzed the 
set of samples shown in Table 1 in a focus forming neutralization assay (Table 2). Each sample was analyzed for five incubation times $(8,9,10,12,14 \mathrm{~h})$ in three independent experiments performed on different days. Variation in neutralization titers was generally low both between the different time points as well among the replicates. The $10 \mathrm{~h}$ time point was chosen as optimal for further experiments as it showed maximum infection without clustering and therefore allowed easy, reproducible automatic counting (Figure 1, Supplementary Figure S1). Finally, to determine interassay variability we analyzed sample P3 in a 1:64 dilution in 18 independent experiments. Figure 1c shows a high reproducibility of the assay.

a.

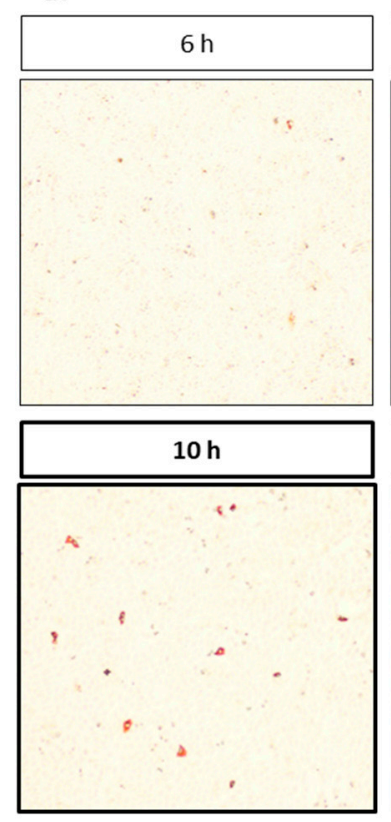

b.

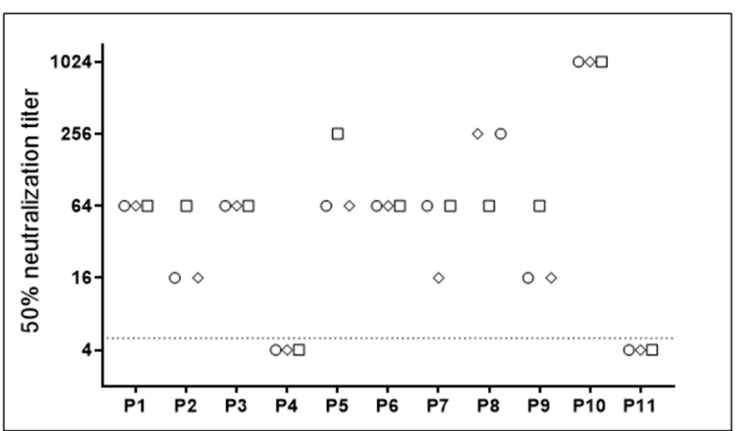

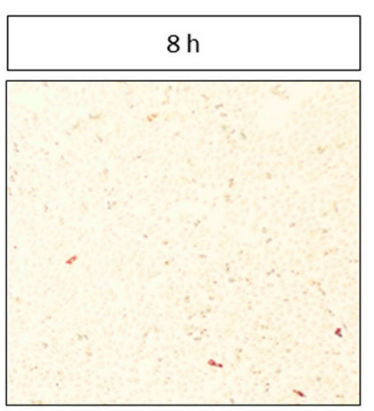
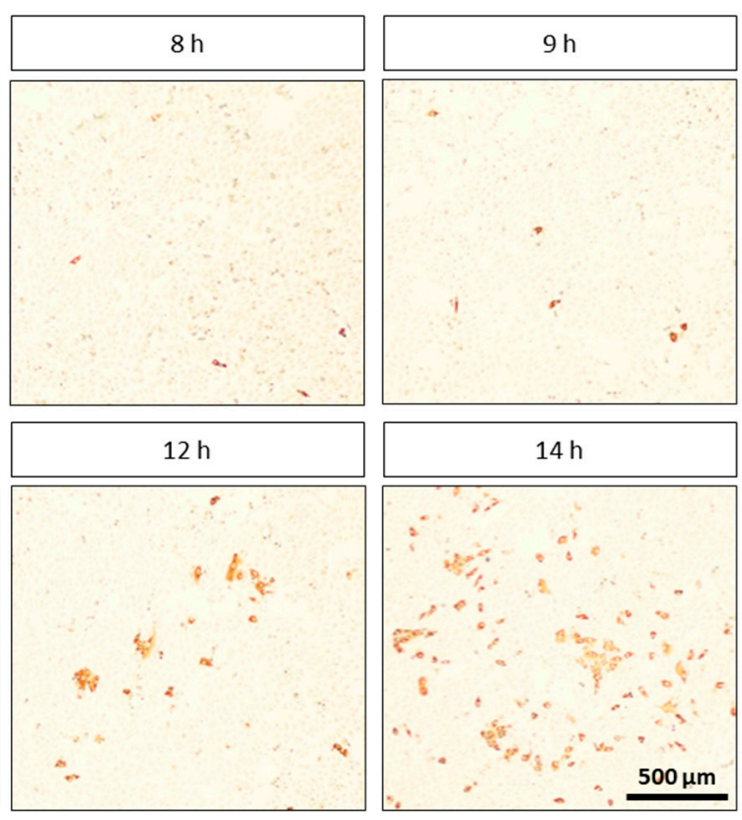

c.

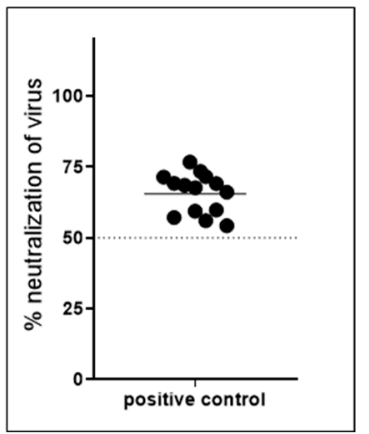

Figure 1. Focus forming neutralization assay. (a) Vero-TMPRSS2 cells were infected with replication competent SARS-CoV-2 virus, fixed after indicated incubation times and stained with the serum of a SARS-CoV-2 convalescent patient. Representative microscopic pictures are shown. (b) Neutralization titers for a set of 11 samples were determined using replication competent SARS-CoV-2 in a focus forming assay. Cells were fixed and stained after $10 \mathrm{~h}$ incubation and neutralization titers were determined using ImmunoSpot S6 Ultra-V reader and CTL analyzer BioSpot ${ }^{\circledR} 5.0$ software (CTL Europe $\mathrm{GmbH}$, Bonn, Germany). The data presented are the result of three independent experiments. Each experiment is represented by a different symbol. Dotted line indicates detection limit for the assay; titers $\leq 1: 4$ were regarded as negative. (c) Low interassay variability and high reproducibility of the assay was demonstrated by analyzing neutralization titers of a positive control (P3, 1:64 dilution) in 18 independent experiment. Percent neutralization was calculated relative to virus only wells. Shown are individual replicates and mean. 
Table 2. Neutralization titers for focus forming assay after different infection times.

\begin{tabular}{|c|c|c|c|c|c|c|c|c|c|c|c|c|c|c|c|}
\hline \multirow{2}{*}{$\frac{\text { Patient }}{\text { P1 }}$} & \multicolumn{3}{|c|}{$8 \mathrm{~h}$} & \multicolumn{3}{|c|}{$9 \mathrm{~h}$} & \multicolumn{3}{|c|}{$10 \mathrm{~h}$} & \multicolumn{3}{|c|}{$12 \mathrm{~h}$} & \multicolumn{3}{|c|}{$14 \mathrm{~h}$} \\
\hline & 64 & 64 & 256 & 64 & 256 & 64 & 64 & 64 & 64 & 64 & 64 & 64 & 256 & 256 & 64 \\
\hline P2 & 16 & 16 & 16 & 16 & 64 & 16 & 16 & 64 & 16 & 16 & 16 & 16 & 16 & 64 & 64 \\
\hline P3 & 64 & 256 & 64 & 64 & 64 & 64 & 64 & 64 & 64 & 64 & 64 & 64 & 256 & 256 & 16 \\
\hline P4 & 4 & 4 & 4 & 4 & 4 & 4 & 4 & 4 & 4 & 4 & 4 & 4 & 4 & 4 & 4 \\
\hline P5 & 64 & 64 & 64 & 64 & 16 & 64 & 64 & 256 & 64 & 64 & 64 & 256 & 64 & 256 & 16 \\
\hline P6 & 16 & 64 & 64 & 64 & 64 & 64 & 64 & 64 & 64 & 256 & 64 & 64 & 64 & 256 & 64 \\
\hline P7 & 16 & 64 & 16 & 64 & 64 & 664 & 64 & 64 & 16 & 64 & 64 & 16 & 64 & 64 & 16 \\
\hline P8 & 64 & 256 & 256 & 256 & 64 & 64 & 256 & 64 & 256 & 64 & 256 & 256 & 256 & 1024 & 256 \\
\hline P9 & 16 & 64 & 16 & 16 & 64 & 64 & 16 & 64 & 16 & 64 & 64 & 16 & 16 & 64 & 16 \\
\hline P10 & 256 & 1024 & 1024 & 1024 & 1024 & 1024 & 1024 & 1024 & 1024 & 1024 & 1024 & 1024 & 1024 & 1024 & 1024 \\
\hline P11 & 4 & 4 & 4 & 4 & 4 & 4 & 4 & 4 & 4 & 4 & 4 & 4 & 4 & 4 & 4 \\
\hline
\end{tabular}

\subsection{TCID50 Assay Using Replication Competent SARS-CoV-2}

As a second assay using replication competent SARS-CoV-2 we performed a TCID 50 -based assay. In a first step, we analyzed cytopathic effects (CPE) of our SARS-CoV-2 isolate on Vero cells expressing TMPRSS2 or parental Vero cells, which was not very prominent (Figure 2a). To enhance susceptibility of cells to SARS-CoV-2 replication we overexpressed ACE2, the receptor for SARS-CoV-2, using a lentiviral vector. The resulting Vero-TMPRSS2/ACE2 cells stably overexpressed TMPRSS2 and ACE2 simultaneously via one lentiviral vector encoding TMPRSS2 and a blasticidin resistance cassette and a second one encoding ACE2 and a hygromycin resistance cassette. Cells were selected with blasticidin and hygromycin to maintain expression of both transgenes. While parental Vero cells only expressed marginal amounts of ACE2, stable Vero-TMPRSS2/ACE2 cells expressed high levels of ACE2 (Supplementary Figure S2a). Overexpression of ACE2 indeed made Vero-TMPRSS2 cells more susceptible to SARS-CoV-2 infection as the same virus had $\mathrm{a} \approx 1 \log$ increased titer on Vero cells overexpressing TMPRSS2 and ACE2 compared to Vero cells only expressing TMPRSS2 when titrating the virus in a focus forming assay (data not shown). In parallel, the CPE on Vero-TMPRSS2/ACE2 cells was also drastically enhanced (Figure 2a).

a.

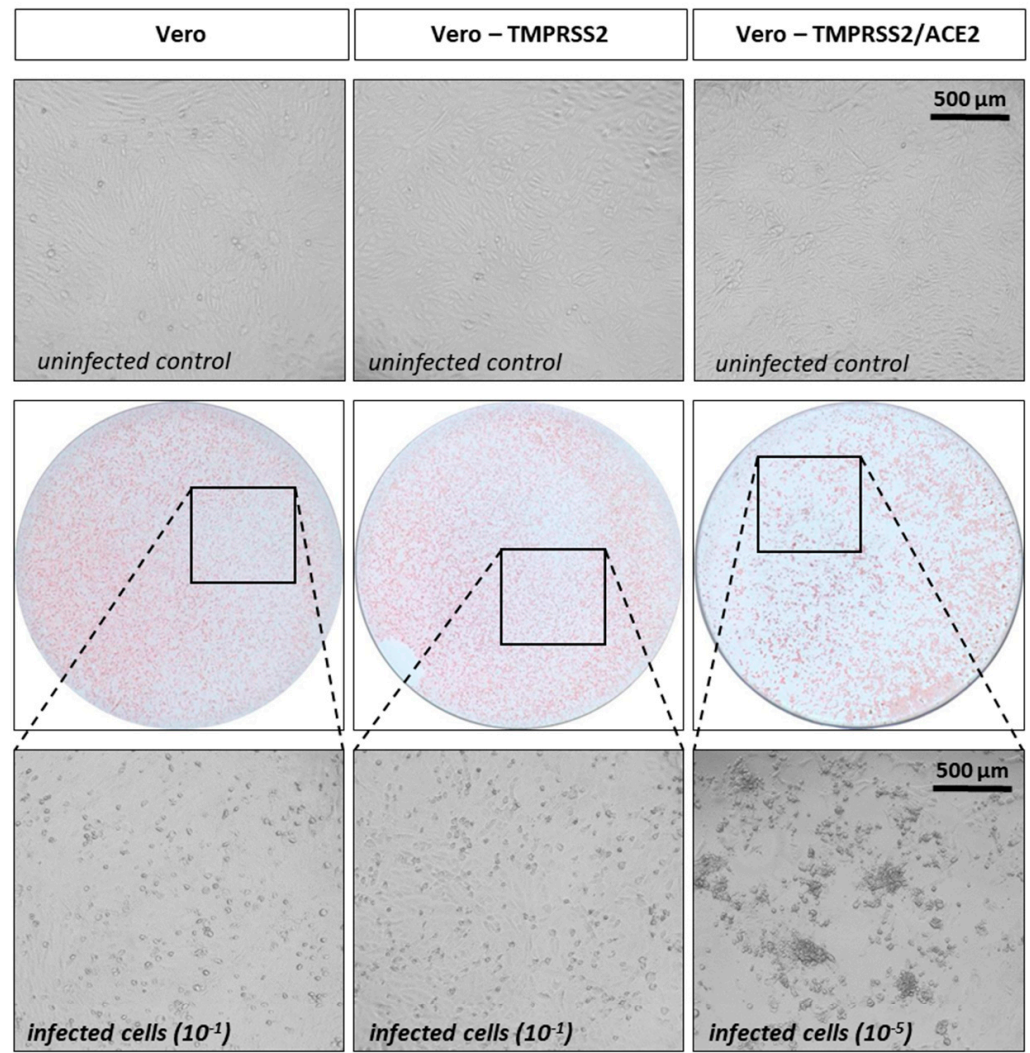

Figure 2. Cont. 
b.

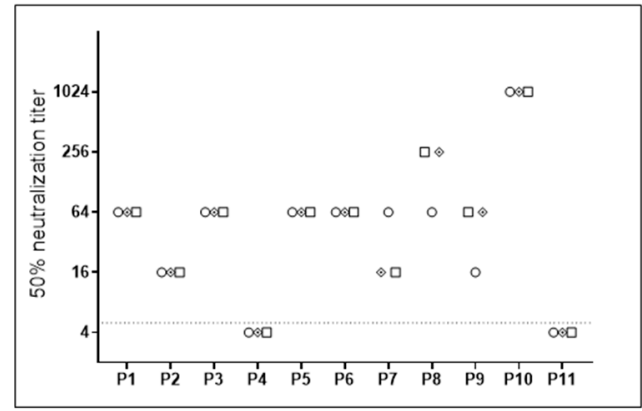

Figure 2. $\mathrm{TCID}_{50}$ neutralization assay. (a) Parental Vero cells, Vero cells expressing TMPRSS2, or Vero cells expressing TMPRSS2 and ACE2 were infected with serial dilutions of SARS-CoV-2. A total of $48 \mathrm{~h}$ after infection CPE was analyzed under the microscope and representative microscopic pictures are shown. Additionally, cells were fixed and stained with the serum of a SARS-CoV-2 convalescent patient. Upper panel: noninfected cells; middle panel: immunostaining $\left(10^{-1}\right.$ dilution for Vero and Vero-TMPRSS2 cells, $10^{-5}$ dilution for Vero-TMPRSS2/ACE2 cells); lower panel: higher magnification of middle panel to evaluate CPE. (b) Neutralization titers for a set of 11 samples were determined using replication competent SARS-CoV-2 in a TCID 50 assay. Neutralization titers of three independent experiments are displayed with different symbols. Dotted line indicates detection limit for the assay; titers $\leq 1: 4$ were regarded as negative.

For the neutralization assay we incubated $100 \mathrm{TCID}_{50}$ of SARS-CoV-2 with different dilutions of patient sera for $1 \mathrm{~h}$, infected Vero-TMPRSS2/ACE2 cells, and after 2 days analyzed CPEs. We determined the $50 \%$ neutralization $\left(\mathrm{TCID}_{50}\right.$ ) titers for our standard set of samples in three independent experiments using the TCID 50 -based assay (Figure 2b). As before for the focus forming assay, interassay variability was low for the $\mathrm{TCID}_{50}$-based assay.

\subsection{VSV-Based Assay}

Although both assays using replication competent SARS-CoV-2 produced highly reproducible results, their need of aBSL-3 laboratory limits applicability. Therefore, we established assays using replication defective VSV $\Delta \mathrm{G}$ or lentiviral particles pseudotyped with SARS-CoV-2 S, which can be performed under BSL-2 conditions.

In a first step, we compared titers for replication defective VSV $\Delta G$ particles pseudotyped with SARS-CoV-2 full length or C-terminally truncated spike protein of SARS-CoV2and found higher titers for the C-terminally truncated variant (Supplementary Figure S3). To facilitate production of replication defective VSV $\Delta$ G particles pseudotyped with SARSCoV-2 $\mathrm{S}$ we next established a BHK-21 cell line stably expressing the C-terminally truncated $\mathrm{S}$ variant $(\mathrm{S} \Delta 18)$, which has previously also by others been shown to produce better titers for lentiviral and VSV-based particles [13]. We used these cells to produce VSV $\Delta G$ particles with different reporter genes, GFP or luciferase. VSV $\Delta$ G-GFP-S particles, expressing GFP as a marker gene, were titrated on different cell lines (Figure 3a, Supplementary Figure S2b). We used wild type 293T with a low or absent expression of ACE2 and hamster BHK-21 cells, which do not express human ACE2 and are consequently not susceptible to SARS$\mathrm{CoV}-2$ infection. The same cells were also transduced with lentiviral vectors to overexpress human ACE2, the receptor for SARS-CoV-2, either in combination with a blasticidin (B) or a hygromycin $(\mathrm{H})$ resistance cassette. For the African green monkey cell line Vero we used three different variants; (i) parental cells, (ii) cells overexpressing TMPRSS2, for which an enhanced infectability has been shown previously, and (iii) a newly generated variant simultaneously overexpressing TMPRSS2 and ACE2. While parental 293T and especially BHK-21 cells were only poorly infected by S pseudotyped VSV particles, overexpression of ACE2 strongly enhanced infection. As seen in replication competent SARS-CoV-2 virus, overexpression of ACE2 enhanced susceptibility of Vero cells expressing TMPRSS2 also for VSV $\Delta$ G-S particles. Infectability of cells with SARS-CoV-2 correlated with ACE2 expression (Supplementary Figure S2). 
a.

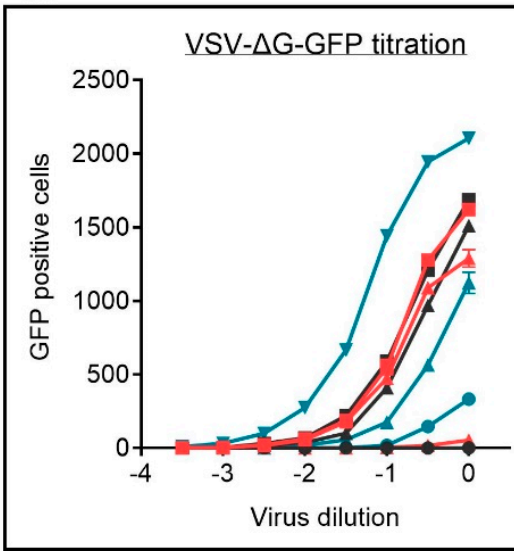

C.

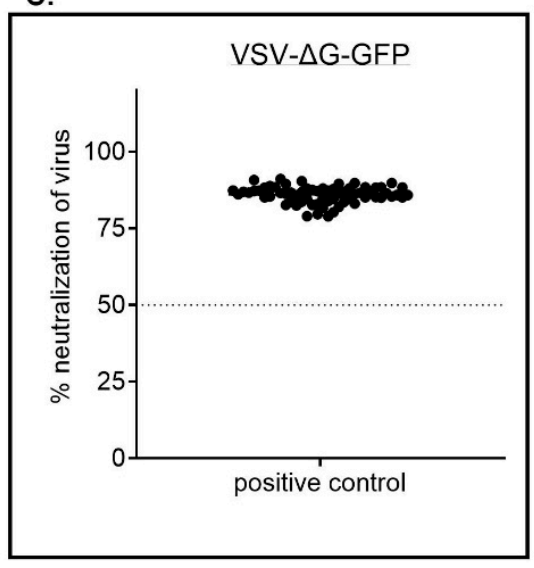

b.

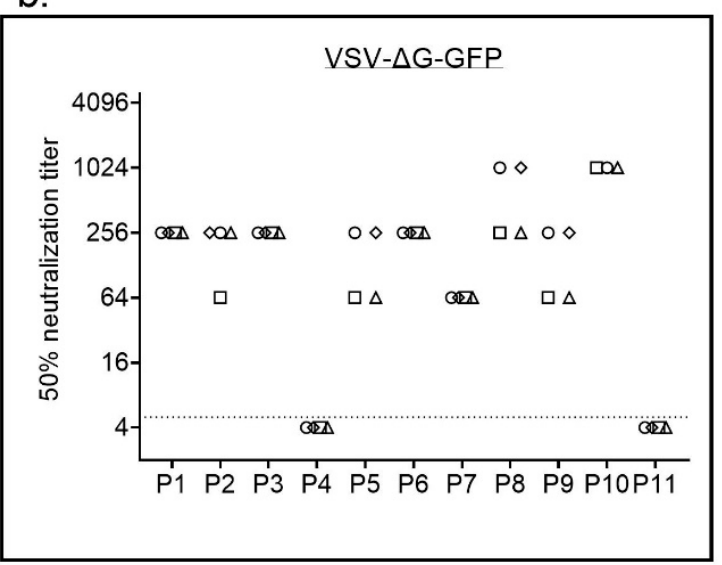

d.

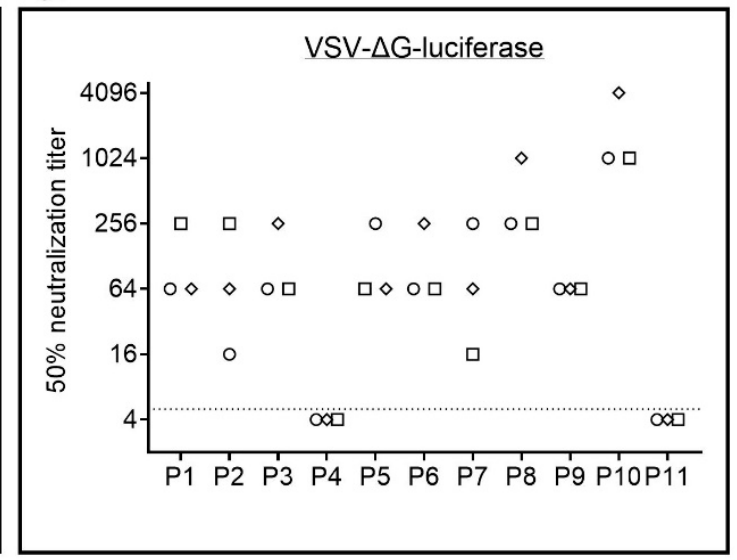

Figure 3. Vesicular stomatitis virus (VSV)-based neutralization assay. (a) VSV $\Delta$ G-GFP-S particles were titrated on parental 293T, BHK21, and Vero cells or on cell lines with stable ACE2 overexpression. For 293T and BHK-21 cells two different lentiviral vectors were used for ACE2 overexpression, either with a blasticinin resistance (B) or a hygromycin resistance cassette $(\mathrm{H})$. A total of $16 \mathrm{~h}$ after infection, the number of GFP positive cells was determined using an immunospot reader. Shown are mean \pm SEM of duplicate samples from one representative of two independent experiments. (b) Neutralization titers for a set of 11 samples were determined using a GFP encoding VSV $\Delta$ G-S variant. Each sample was analyzed in four independent experiments. Each experiment is represented by a different symbol. Neutralization titers $\leq 1: 4$ are regarded negative. (c) A positive control (P3 in a 1:64 dilution) was analyzed using the VSV $\Delta$ G-GFP-S virus on 66 independent plates. Percent neutralization was calculated relative to virus only wells. Shown are individual replicates and mean. (d) Neutralization titers for a set of 11 samples were determined using a luciferase encoding VSV $\Delta$ G-S variant. Each sample was analyzed in three independent experiments. Each experiment is represented by a different symbol. Dotted line in $b+d$ indicate threshold for neutralizing antibody titers, i.e., titers $\leq 1: 4$ are regarded as negative. Dotted line in c indicates $50 \%$ neutralization.

In a next step, we compared VSV $\Delta \mathrm{G}$ variants encoding different reporter genes, GFP or luciferase, for the neutralization assay. All assays were performed on 293T-ACE2 cells. Automatic counting of GFP positive cells using an immunospot reader worked well (Supplementary Figure S4). GFP and luciferase encoding viruses resulted in similar neutralization titers with a low interassay variability (Figure 3b,d, Supplementary Figure S5). For the GFP encoding virus we analyzed patient P3 in a 1:64 dilution in 66 independent experiments and also saw a high reproducibility of the assay (Figure 3c).

\subsection{Lentiviral Vector Based Assay}

As a second BSL-2 compatible system we used lentiviral particles pseudotyped with SARS-CoV-2 S expressing GFP as reporter. Again, a C-terminally truncated S variant was used to increase virus titers. Patient sera were preincubated with the virus and subsequently 293T cells expressing ACE2 were infected. As for the VSV $\Delta$ G, GFP positive cells could be 
counted with the immunospot reader. However, automatic counting was more difficult as infected cells divided (Supplementary Figure S6). The same set of patients as before was analyzed for the lentiviral particle-based SARS-CoV-2 neutralization assay in three independent experiments (Figure 4). The titers obtained with the lentiviral particles were more variable compared to the other systems, especially for weakly positive samples such as P2 and P7.

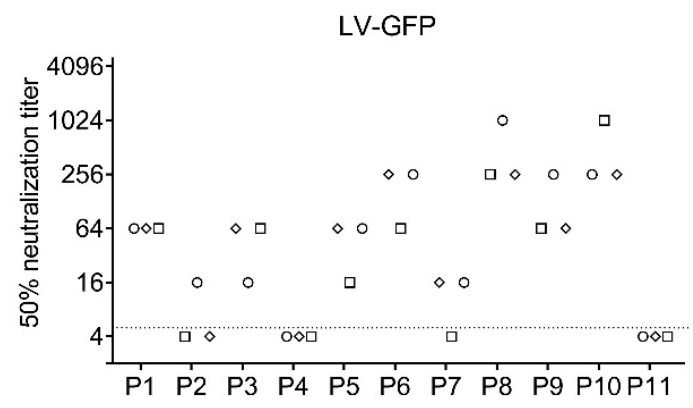

Figure 4. Lentiviral particle-based neutralization assay. Lentiviral particles encoding GFP were pseudotyped with SARS-CoV-2 S. Neutralization titers for a set of 11 samples were determined. Each sample was analyzed in three independent experiments. Each experiment is represented by a different symbol. Dotted line indicates detection limit for the assay; titers $\leq 1: 4$ were regarded as negative.

\subsection{Comparison of Assays}

We showed that each of the four SARS-CoV-2 neutralization assays we used was highly reproducible and had a low interassay variability. Neutralizing antibody titer obtained with each of the assays correlated well with each of the immunoassays for binding antibodies (Figure 5a, Table 3). The most interesting question in this study was how comparable the different neutralizing antibody assays were. As different labs use different assays to determine SARS-CoV-2 neutralizing antibody titers it is crucial to compare neutralization titers obtained with all assays using a standard set of samples. Therefore, in a last step, we compared neutralization titers for our standard patient samples for all assays (Figure $5 b$ ). We correlated each assay with the other three assays and determined Spearman's $r$ (Table 4). Neutralization titers for all four assays correlated well with each other with a tendency for lower correlations with the lentiviral particle-based assay. Regarding sensitivity all assays seemed to perform equally well with a consistent detection even of samples with low levels of binding antibodies.

Table 3. Comparison binding antibody titer and neutralization titer.

\begin{tabular}{|c|c|c|c|c|}
\hline \multicolumn{2}{|c|}{ Comparison } & \multirow{2}{*}{$\begin{array}{c}\text { Spearman's r } \\
0.7558\end{array}$} & \multirow{2}{*}{$\begin{array}{c}\mathbf{9 5} \% \text { Confidence Interval } \\
0.2664-0.9354\end{array}$} & \multirow{2}{*}{$\frac{p \text { Value }^{\S}}{0.0093}$} \\
\hline Anti-S IgA & Focus forming & & & \\
\hline Anti-S IgA & $\mathrm{TCID}_{50}$ & 0.8674 & $0.5435-0.9665$ & 0.0010 \\
\hline Anti-S IgA & VSV-GFP & 0.7927 & $0.3498-0.9460$ & 0.0053 \\
\hline Anti-S IgA & Lentiviral particle & 0.8513 & $0.4985-0.9622$ & 0.0015 \\
\hline Anti-S IgG & Focus forming & 0.9033 & $0.6507-0.9759$ & 0.0003 \\
\hline Anti-S IgG & $\mathrm{TCID}_{50}$ & 0.9654 & $0.8632-0.9916$ & $<0.0001$ \\
\hline Anti-S IgG & VSV-GFP & 0.9586 & $0.8382-0.9899$ & $<0.0001$ \\
\hline Anti-S IgG & Lentiviral particle & 0.9291 & $0.7344-0.9825$ & $<0.0001$ \\
\hline Anti-N IgG & Focus forming & 0.8526 & $0.5022-0.9625$ & 0.0014 \\
\hline Anti-N IgG & $\mathrm{TCID}_{50}$ & 0.9420 & $0.7788-0.9858$ & $<0.0001$ \\
\hline Anti-N IgG & VSV-GFP & 0.9586 & $0.8382-0.9899$ & $<0.0001$ \\
\hline Anti-N IgG & Lentiviral particle & 0.9520 & $0.8141-0.9883$ & $<0.0001$ \\
\hline
\end{tabular}

$\S$ two-tailed. 
a.

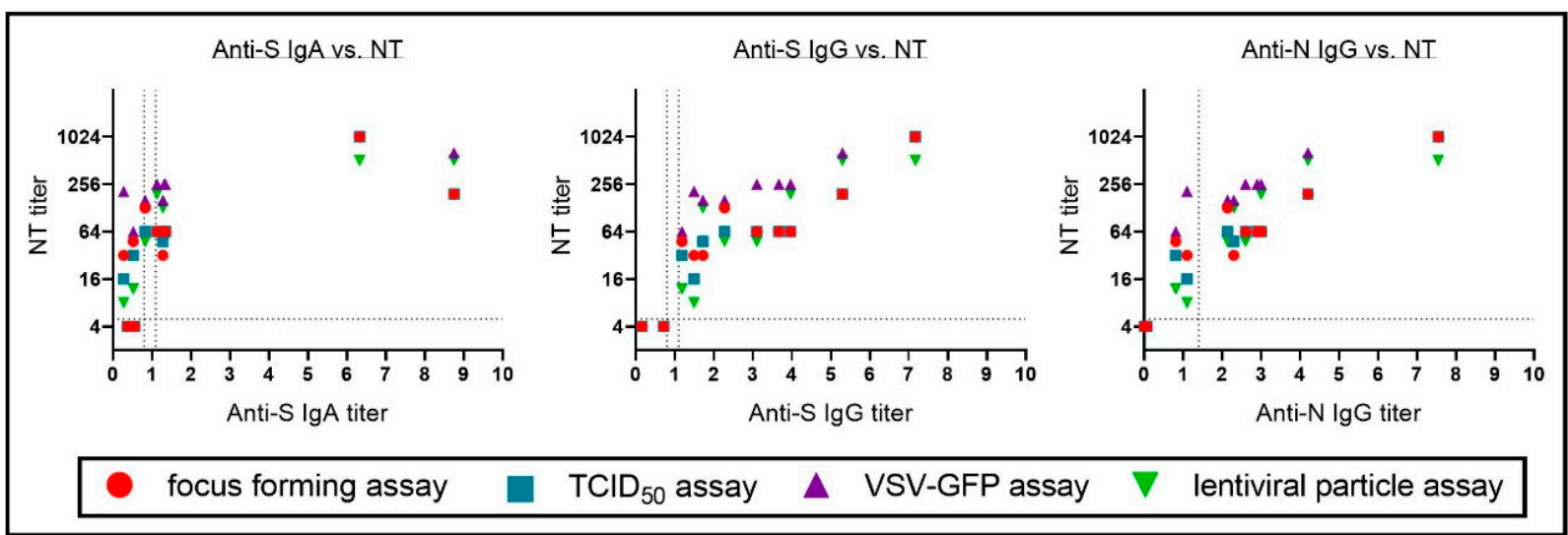

b.

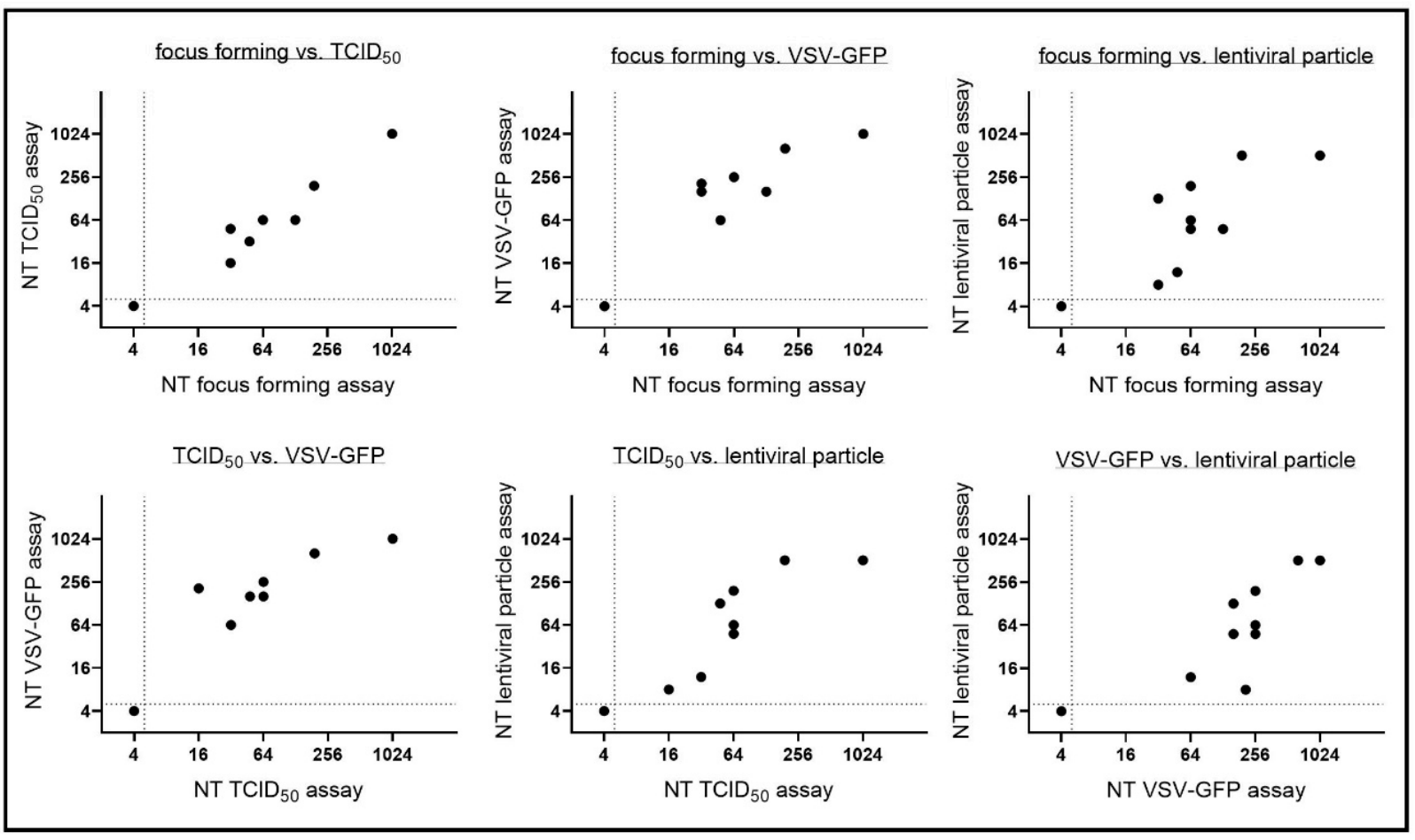

Figure 5. Neutralization titers for the different assays correlate well with each other and with binding antibody titers. For neutralization assays mean neutralizing titers of three (focus forming, TCID $_{50}$, and lentiviral particle assay) or four independent experiments (VSV assay) were calculated. (a) Correlation of neutralizing antibody titers and binding antibodies (anti-S IgA, anti-S IgG, and anti-N IgG) were determined. (b) Neutralizing antibody titers determined in each assay were correlated to titers in all three other neutralizing antibody assays. Dotted lines indicate detection limit for the different assays; neutralization assay $\leq 1: 4$ negative, anti-S IgA and anti-S IgG OD $<0.8$ negative, OD 0.8-1.1 borderline, OD $>1.1$ positive, anti-N IgG RLU > 1.4 positive. Some of the dots in the blots represent multiple samples.

Table 4. Comparison of neutralization assays.

\begin{tabular}{ccccc}
\hline \multicolumn{2}{c}{ Comparison } & Spearman's $\mathbf{r}$ & 95\% Confidence Interval & $p$ Value \\
\hline Focus forming & TCID $_{50}$ & 0.9693 & $0.8782-0.9926$ & $<0.0001$ \\
Focus forming & VSV-GFP & 0.8388 & $0.4650-0.9588$ & 0.0019 \\
Focus forming & Lentiviral particle & 0.8121 & $0.396-0.9514$ & 0.0035 \\
TCID50 & VSV-GFP & 0.9055 & $0.6576-0.9765$ & 0.0002 \\
TCID & Lentiviral particle & 0.9063 & $0.6600-0.9767$ & 0.0003 \\
VSV-GFP & Lentiviral particle & 0.8515 & $0.4992-0.9622$ & 0.0015 \\
\hline
\end{tabular}

\& two-tailed. 


\section{Discussion}

In the present study we established and compared four different SARS-CoV-2 neutralization assays, a focus forming and a $\mathrm{TCID}_{50}$-based assay using replication competent SARS-CoV-2, and two pseudovirus assays, one based on VSV and the other based on lentiviral particles. As many different SARS-CoV-2 neutralization assays are commonly used it is very important to cross reference these different assays. This will allow a better comparison of studies performed in different labs. We found that results for all four neutralization assays correlated well with each other. Similar to others, we also found a correlation of binding IgG and neutralizing antibodies.

Pseudotyped virus assays have the advantage that they do not require BSL-3 laboratories and are therefore easier to establish and allow higher throughput. When analyzing the efficacy of vaccine candidates based on chimeric VSV variants there is a potential risk of cross-reactivity of antibodies against the vaccine vector with the pseudotyped VSV used for the neutralization assay. However, as the pseudovirus particles lack an endogens envelope glycoprotein and SARS-CoV-2 spike protein is the only viral protein responsible for neutralization this cross reactivity is highly unlikely.

Similar to others we also saw an increased titer for VSV-based particles pseudotyped with a C-terminally truncated version of the spike protein compared to a full-length spike (data not shown) $[13,18]$. This is in accordance with previous reports for other coronaviruses showing that truncation of spike protein enhances incorporation into viral particles [22,23]. The C-terminus of coronavirus spike protein contains an ER retention signal, which is most likely the reason that its deletion enhances transport of spike protein to the cell surface and incorporation into viral particles that bud from the cell surface such as lentiviruses and VSV.

Pseudotyped viruses are also a valid tool for identification of alternate receptors of SARS-CoV-2 or cofactors enhancing infection without the need of a BSL-3 facility. Similar to others, we observed an increased susceptibility of cells for the SARS-CoV-2 spike pseudotyped VSV when human ACE2 was overexpressed. Recently, also TMPRSS2 and Neuropilin-1 have been described as cofactors enhancing SARS-CoV-2 entry [16,24].

In our study, both assays using pseudotyped viruses produced reproducible results, which correlated well with binding antibody titers obtained in the immunoassays and neutralizing antibody titers determined with replication competent SARS-CoV-2. Patient plasma samples were reproducibly positive or negative in all assays, with the exception of the lentiviral particle-based assay, where two patients gave positive neutralization titers in some experiments and negatives in others. In general, we saw a slightly lower correlation of the other test systems with the assay using lentiviral particles than for the VSV-based assay. This might be explained by the readout of the number of GFP positive cells using an immunospot reader. As lentiviral particles, in contrast to the VSV particles, integrate, the GFP signal is maintained upon cell division leading to clusters of GFP positive cells for lentiviral particles. As we also saw for the replication competent SARS-CoV-2 virus, clusters of cells are more difficult to count using automatic counting in the immunospot reader.

Remarkably, the two patients with discrepant results regarding neutralizing antibodies (P2 and P7) had also discrepant results in the immunoassays and were positive for anti-S IgG but negative for anti-N IgG indicating a weak immunity to SARS-CoV-2 in these patients. In our previous epidemiological study in an Austrian SARS-CoV-2 hotspot the majority of patients with discrepant immunoassays were positive for neutralizing antibodies [11]. In our current study also the VSV-based assay had a high sensitivity in patients with discrepant immunoassay results.

A further advantage of assays using pseudotyped viruses is that different variants of SARS-CoV-2 spike protein can easily be used for pseudotyping and therefore can be compared regarding their neutralization sensitivity. Mutations attributed to resistance to certain SARS-CoV-2 monoclonal neutralizing antibodies have recently been described $[25,26]$. A panel of different pseudotyped VSV variants could be used to map resistance in human plasma samples. This approach could also be helpful to analyze if different vaccine ap- 
proaches, e.g., full length spike protein versus single domains, induce different types of neutralizing antibody responses. Resistance to certain neutralizing antibodies as well as generally low antibody titers could allow reinfections, as recently described [8,9]. Further studies will be needed to determine threshold titers of neutralizing antibodies needed for protection. However, all four assays described here result in robust and reproducible neutralization titers and are therefore good candidates for further studies.

Supplementary Materials: The following are available online at https://www.mdpi.com/2076 -393X/9/1/13/s1, Figure S1: Exemplary wells for focus forming assay after counting using an immunospot reader, Figure S2: ACE2 expression determines infectability of cells with VSV $\Delta$ G-S particles, Figure S3: A C-terminally spike variant produces higher titers of pseudotyped VSV vectors compared to full length spike, Figure S4. Exemplary wells for VSV-based neutralization assay after counting using an immunospot reader, Figure S5. Inhibition curves for VSV-based neutralization assay, Figure S6. Exemplary wells for lentiviral particle-based neutralization assay after counting using an immunospot reader, Figure S7. Inhibition curves for lentiviral particle-based neutralization assay.

Author Contributions: Conceptualization, D.v.L. and J.K.; Investigation, L.R., A.R., A.F. and J.K.; Resources, A.V. and W.B.; Supervision, J.K.; Visualization, L.R., A.R. and J.K.; Writing-original draft, L.R., A.R. and J.K.; Writing - review and editing, A.F., A.V., W.B. and D.v.L. All authors have read and agreed to the published version of the manuscript.

Funding: This research received no external funding.

Institutional Review Board Statement: The study was conducted according to the guidelines of the Declaration of Helsinki, and approved by the Ethics Committee of the Medical University of Innsbruck (EC number: 1100/2020, 19.04.2020).

Informed Consent Statement: Informed consent was obtained from all subjects involved in the study.

Data Availability Statement: The data presented in this study are available in the article and supplementary material.

Acknowledgments: We thank Csaba Merschdorf and Attila L. Edelmann (CTL Europe GmbH, Bonn, Germany) for excellent technical support.

Conflicts of Interest: The authors declare no conflict of interest.

\section{References}

1. World Health Organization. WHO Coronavirus Disease (COVID-19) Dashboard. Available online: https:// covid19.who.int (accessed on 22 December 2020).

2. FIND. SARS-CoV-2 Diagnostic Pipeline. Available online: https://www.finddx.org/covid-19/pipeline/ (accessed on 22 December 2020).

3. Meyer, B.; Torriani, G.; Yerly, S.; Mazza, L.; Calame, A.; Arm-Vernez, I.; Zimmer, G.; Agoritsas, T.; Stirnemann, J.; Spechbach, H.; et al. Validation of a commercially available SARS-CoV-2 serological immunoassay. Clin. Microbiol. Infect. 2020, 26, 1386-1394. [CrossRef] [PubMed]

4. Grzelak, L.; Temmam, S.; Planchais, C.; Demeret, C.; Tondeur, L.; Huon, C.; Guivel-Benhassine, F.; Staropoli, I.; Chazal, M.; Dufloo, J.; et al. A comparison of four serological assays for detecting anti-SARS-CoV-2 antibodies in human serum samples from different populations. Sci. Transl. Med. 2020, 12, eabc3103. [CrossRef] [PubMed]

5. Zhao, J.; Yuan, Q.; Wang, H.; Liu, W.; Liao, X.; Su, Y.; Wang, X.; Yuan, J.; Li, T.; Li, J.; et al. Antibody responses to SARS-CoV-2 in patients of novel coronavirus disease 2019. Clin. Infect. Dis. 2020, 71, 2027-2034. [CrossRef] [PubMed]

6. Long, Q.X.; Liu, B.Z.; Deng, H.J.; Wu, G.C.; Deng, K.; Chen, Y.K.; Liao, P.; Qiu, J.F.; Lin, Y.; Cai, X.F.; et al. Antibody responses to SARS-CoV-2 in patients with COVID-19. Nat. Med. 2020, 26, 845-848. [CrossRef] [PubMed]

7. Kellam, P.; Barclay, W. The dynamics of humoral immune responses following SARS-CoV-2 infection and the potential for reinfection. J. Gen. Virol. 2020, 101, 791-797. [CrossRef] [PubMed]

8. Gupta, V.; Bhoyar, R.C.; Jain, A.; Srivastava, S.; Upadhayay, R.; Imran, M.; Jolly, B.; Divakar, M.K.; Sharma, D.; Sehgal, P.; et al. Asymptomatic reinfection in two healthcare workers from India with genetically distinct SARS-CoV-2. Clin. Infect. Dis. 2020. [CrossRef]

9. To, K.K.; Hung, I.F.; Ip, J.D.; Chu, A.W.; Chan, W.M.; Tam, A.R.; Fong, C.H.; Yuan, S.; Tsoi, H.W.; Ng, A.C.; et al. COVID-19 re-infection by a phylogenetically distinct SARS-coronavirus-2 strain confirmed by whole genome sequencing. Clin Infect Dis. 2020. [CrossRef]

10. Peterhoff, D.; Gluck, V.; Vogel, M.; Schuster, P.; Schutz, A.; Neubert, P.; Albert, V.; Frisch, S.; Kiessling, M.; Pervan, P.; et al. A highly specific and sensitive serological assay detects SARS-CoV-2 antibody levels in COVID-19 patients that correlate with neutralization. Infection 2020. [CrossRef] 
11. Knabl, L.; Mitra, T.; Kimpel, J.; Roessler, A.; Volland, A.; Walser, A.; Ulmer, H.; Pipperger, L.; Binder, S.C.; Riepler, L.; et al. High SARS-CoV-2 Seroprevalence in Children and Adults in the Austrian Ski Resort Ischgl. medRxiv 2020. [CrossRef]

12. Amanat, F.; White, K.M.; Miorin, L.; Strohmeier, S.; McMahon, M.; Meade, P.; Liu, W.C.; Albrecht, R.A.; Simon, V.; MartinezSobrido, L.; et al. An In Vitro Microneutralization Assay for SARS-CoV-2 Serology and Drug Screening. Curr. Protoc. Microbiol. 2020, 58, e108. [CrossRef]

13. Schmidt, F.; Weisblum, Y.; Muecksch, F.; Hoffmann, H.H.; Michailidis, E.; Lorenzi, J.C.C.; Mendoza, P.; Rutkowska, M.; Bednarski, E.; Gaebler, C.; et al. Measuring SARS-CoV-2 neutralizing antibody activity using pseudotyped and chimeric viruses. J. Exp. Med. 2020, 217, 11. [CrossRef] [PubMed]

14. Crawford, K.H.D.; Eguia, R.; Dingens, A.S.; Loes, A.N.; Malone, K.D.; Wolf, C.R.; Chu, H.Y.; Tortorici, M.A.; Veesler, D.; Murphy, M.; et al. Protocol and Reagents for Pseudotyping Lentiviral Particles with SARS-CoV-2 Spike Protein for Neutralization Assays. Viruses 2020, 12, 513. [CrossRef] [PubMed]

15. Zettl, F.; Meister, T.L.; Vollmer, T.; Fischer, B.; Steinmann, J.; Krawczyk, A.; V'Kovski, P.; Todt, D.; Steinmann, E.; Pfaender, S.; et al. Rapid Quantification of SARS-CoV-2-Neutralizing Antibodies Using Propagation-Defective Vesicular Stomatitis Virus Pseudotypes. Vaccines 2020, 8, 386. [CrossRef] [PubMed]

16. Hoffmann, M.; Kleine-Weber, H.; Schroeder, S.; Kruger, N.; Herrler, T.; Erichsen, S.; Schiergens, T.S.; Herrler, G.; Wu, N.H.; Nitsche, A.; et al. SARS-CoV-2 Cell Entry Depends on ACE2 and TMPRSS2 and Is Blocked by a Clinically Proven Protease Inhibitor. Cell 2020, 181, 271-280. [CrossRef]

17. Nie, J.; Li, Q.; Wu, J.; Zhao, C.; Hao, H.; Liu, H.; Zhang, L.; Nie, L.; Qin, H.; Wang, M.; et al. Establishment and validation of a pseudovirus neutralization assay for SARS-CoV-2. Emerg. Microbes Infect. 2020, 9, 680-686. [CrossRef]

18. Hoffmann, M.; Kleine-Weber, H.; Pohlmann, S. A Multibasic Cleavage Site in the Spike Protein of SARS-CoV-2 Is Essential for Infection of Human Lung Cells. Mol. Cell. 2020, 78, 779-784 e775. [CrossRef]

19. Hermann, F.G.; Egerer, L.; Brauer, F.; Gerum, C.; Schwalbe, H.; Dietrich, U.; von Laer, D. Mutations in gp120 contribute to the resistance of human immunodeficiency virus type 1 to membrane-anchored C-peptide maC46. J. Virol. 2009, 83, 4844-4853. [CrossRef]

20. Hoffmann, M.; Wu, Y.J.; Gerber, M.; Berger-Rentsch, M.; Heimrich, B.; Schwemmle, M.; Zimmer, G. Fusion-active glycoprotein G mediates the cytotoxicity of vesicular stomatitis virus M mutants lacking host shut-off activity. J. Gen. Virol. 2010, 91, 2782-2793. [CrossRef]

21. Tober, R.; Banki, Z.; Egerer, L.; Muik, A.; Behmuller, S.; Kreppel, F.; Greczmiel, U.; Oxenius, A.; von Laer, D.; Kimpel, J. VSV-GP: A potent viral vaccine vector that boosts the immune response upon repeated applications. J. Virol. 2014, 88, 4897-4907. [CrossRef]

22. Giroglou, T.; Cinatl, J., Jr.; Rabenau, H.; Drosten, C.; Schwalbe, H.; Doerr, H.W.; von Laer, D. Retroviral vectors pseudotyped with severe acute respiratory syndrome coronavirus S protein. J. Virol. 2004, 78, 9007-9015. [CrossRef]

23. Lin, H.X.; Feng, Y.; Tu, X.; Zhao, X.; Hsieh, C.H.; Griffin, L.; Junop, M.; Zhang, C. Characterization of the spike protein of human coronavirus NL63 in receptor binding and pseudotype virus entry. Virus Res. 2011, 160, 283-293. [CrossRef] [PubMed]

24. Cantuti-Castelvetri, L.; Ojha, R.; Pedro, L.D.; Djannatian, M.; Franz, J.; Kuivanen, S.; van der Meer, F.; Kallio, K.; Kaya, T.; Anastasina, M.; et al. Neuropilin-1 facilitates SARS-CoV-2 cell entry and infectivity. Science 2020, 370, 856-860. [CrossRef] [PubMed]

25. Baum, A.; Fulton, B.O.; Wloga, E.; Copin, R.; Pascal, K.E.; Russo, V.; Giordano, S.; Lanza, K.; Negron, N.; Ni, M.; et al. Antibody cocktail to SARS-CoV-2 spike protein prevents rapid mutational escape seen with individual antibodies. Science 2020, 369, 1014-1018. [CrossRef] [PubMed]

26. Weisblum, Y.; Schmidt, F.; Zhang, F.; DaSilva, J.; Poston, D.; Lorenzi, J.C.; Muecksch, F.; Rutkowska, M.; Hoffmann, H.H.; Michailidis, E.; et al. Escape from neutralizing antibodies by SARS-CoV-2 spike protein variants. eLife 2020, 9, e61312. [CrossRef] 